\title{
An Efficient Copper Catalyst for the Formation of Sulfones from Sulfinic Acid Salts and Aryl Iodides
}

\author{
Jeremy M. Baskin and Zhaoyin Wang* \\ Department of Medicinal Chemistry, Merck Frosst Centre for Therapeutic Research, \\ Pointe-Claire/Dorval, Quebec, Canada H9R 4P8.
}

\section{Supporting Information}

General Considerations: All reactions were carried out in sealed test tubes under a nitrogen atmosphere. $N, N$ '-dimethylethylenediamine was purchased from Aldrich and used without further purification. Copper (I) triflate $\left((\mathrm{CuOTf})_{2} \mathrm{PhH}\right)$ was purchased from Aldrich. All other reagents were commercially available and were used without further purification. All solids were weighed in the air. Flash column chromatography was

performed with Silica gel 60 (230-400 mesh). ${ }^{1} \mathrm{H}$ and ${ }^{13} \mathrm{C}$ NMR were recorded on a Bruker $500 \mathrm{MHz}$ instrument with chemical shifts reported relative to residual deuterated solvent peaks. All spectra were taken in acetone- $\mathrm{d}_{6}$ and, in addition to the multiplet at $2.07 \mathrm{ppm}$ due to acetone, exhibit a strong singlet at $2.88 \mathrm{ppm}$ and a weak singlet at 2.84 ppm due to residual water absorbed by acetone- $\mathrm{d}_{6}$. HPLC analyses were performed on an Alliance Waters 2690 system with a Waters 990 Photodiode Array Detector. All yields reported in this publication refer to isolated yields of compounds estimated to be $>95 \%$ pure as determined by ${ }^{1} \mathrm{H}$ NMR.

General Procedure for the Copper-Catalyzed Coupling of Sulfinic Acid Salts with Aryl Halides.

(a) Used in the case that the aryl iodide is a solid at room temperature

To a sealable tube with an $8 \times 1.5 \mathrm{~mm}$ stir bar was added $0.05 \mathrm{mmol}$ of $(\mathrm{CuOTf})_{2} \mathrm{PhH}$, $1.20 \mathrm{mmol}$ of the sodium sulfinate, and $1.00 \mathrm{mmol}$ of the aryl iodide under an air 
atmosphere. The aperture of the tube was then covered with a rubber septum and a nitrogen atmosphere was established. $N, N^{\prime}$-dimethylethylenediamine $(0.10 \mathrm{mmol})$ and anhydrous DMSO $(1 \mathrm{~mL})$ were added via syringe. The septum was replaced by a teflon coated screw cap and the reaction vessel was placed in a $110 \mathrm{C}$ oil bath. After stirring for approximately $20 \mathrm{~h}$, the reaction mixture was allowed to cool to room temperature, diluted with $10 \mathrm{~mL}$ of ethyl acetate and filtered through a pad of silica gel. The filtrate was washed with water $(2 \times 20 \mathrm{~mL})$ and brine $(1 \times 20 \mathrm{~mL})$, dried over $\mathrm{Na}_{2} \mathrm{SO}_{4}$, filtered through silica gel, and concentrated. In some cases, further purification by flash column chromatography was required to obtain a pure product. In all cases, the yields reported in the publication represent the isolated yield of products of $>95 \%$ purity as determined by ${ }^{1} \mathrm{H}$ NMR.

(b) Used in the case that the aryl iodide is a liquid at room temperature

To a sealable tube with an $8 \times 1.5 \mathrm{~mm}$ stir bar was added $0.05 \mathrm{mmol}$ of $(\mathrm{CuOTf})_{2} \mathrm{PhH}$ and $1.20 \mathrm{mmol}$ of the sodium sulfinate under an air atmosphere. The aperture of the tube was then covered with a rubber septum and a nitrogen atmosphere was established. $N, N^{\prime}$ dimethylethylenediamine $(0.10 \mathrm{mmol})$, the aryl iodide $(1.00 \mathrm{mmol})$, and anhydrous DMSO (1 mL) were added via syringe. The septum was replaced by a teflon coated screw cap and the reaction vessel was placed in a $110 \mathrm{C}$ oil bath. After stirring for approximately $20 \mathrm{~h}$. the reaction mixture was allowed to cool to room temperature, diluted with $10 \mathrm{~mL}$ of ethyl acetate and filtered through a pad of silica gel. The filtrate was washed with water $(2 \times 20 \mathrm{~mL})$ and brine $(1 \times 20 \mathrm{~mL})$, dried over $\mathrm{Na}_{2} \mathrm{SO}_{4}$, filtered through silica gel, and concentrated. In some cases, further purification by flash column chromatography was required to obtain a pure product. In all cases, the yields reported in the publication represent the isolated yield of products of $>95 \%$ purity as determined by ${ }^{1} \mathrm{H}$ NMR.

1-methanesulfonyl-naphthalene (Table 1, Entry 1). General procedure (b) was followed using $25 \mathrm{mg}(0.05 \mathrm{mmol})(\mathrm{CuOTf})_{2} \mathrm{PhH}, 123 \mathrm{mg}(1.20 \mathrm{mmol})$ methanesulfinic acid, sodium salt, 11 L (0.10 mmol) $N, N^{\prime}$-dimethylethylenediamine, and 146 L (1.00 
mmol) 1-iodonaphthalene. The title compound (197 mg, 96\%) was obtained as a white solid. M.P. $102{ }^{\circ} \mathrm{C}$ (lit. $\left.{ }^{1} 102-103{ }^{\circ} \mathrm{C}\right) .{ }^{1} \mathrm{H}$ NMR $\left(500 \mathrm{MHz}\right.$, acetone- $\left.\mathrm{d}_{6}\right)$ : $8.78(\mathrm{~d}, 1 \mathrm{H}, \mathrm{J}=$ $8.7 \mathrm{~Hz}$ ), 8.33 (t, 1H, J = 4.3 Hz), 8.32 (d, 1H, J = 6.3 Hz), 8.16 (d, 1H, J = 8.2), 7.80 (td, $1 \mathrm{H}, \mathrm{J}=8.5,1.4 \mathrm{~Hz}), 7.73-7.77(\mathrm{~m}, 2 \mathrm{H}), 3.30(\mathrm{~s}, 3 \mathrm{H}) .{ }^{13} \mathrm{C}$ NMR $\left(125 \mathrm{MHz}\right.$, acetone- $\left.\mathrm{d}_{6}\right)$ : 137.18, 135.70, 135.06, 130.07, 130.04, 129.32, 129.26, 127.77, 125.42, 124.91, 44.38. $\mathrm{MS}(\mathrm{APCI})^{+} \mathrm{m} / \mathrm{z}$ (rel intens) $207(\mathrm{M}+\mathrm{H}, 35), 128\left(\mathrm{M}-\mathrm{SO}_{2} \mathrm{Me}, 100\right)$.

2-methanesulfonyl-thiophene (Table 1, Entry 2). General procedure (b) was followed using $25 \mathrm{mg}(0.05 \mathrm{mmol})(\mathrm{CuOTf})_{2} \mathrm{PhH}, 123 \mathrm{mg}(1.20 \mathrm{mmol})$ methanesulfinic acid, sodium salt, $11 \mathrm{~L}(0.10 \mathrm{mmol}) N, N^{\prime}$-dimethylethylenediamine, and $110 \mathrm{~L}(1.00 \mathrm{mmol})$ 2-iodothiophene. The title compound (102 mg, 63\%) was obtained as a beige solid after purification by flash column chromatography eluted with 2:1 hexanes:ethyl acetate. M.P. $47{ }^{\circ} \mathrm{C}$ (lit. ${ }^{2} 47{ }^{\circ} \mathrm{C}$ ). ${ }^{1} \mathrm{H}$ NMR $\left(500 \mathrm{MHz}\right.$, acetone- $\left.\mathrm{d}_{6}\right)$ : $8.02(\mathrm{dd}, 1 \mathrm{H}, \mathrm{J}=5.0,1.3 \mathrm{~Hz}$ ), $7.78(\mathrm{dd}, 1 \mathrm{H}, \mathrm{J}=3.8,1.2 \mathrm{~Hz}), 7.28(\mathrm{dd}, 1 \mathrm{H}, \mathrm{J}=5.0,3.8 \mathrm{~Hz}), 3.26(\mathrm{~s}, 3 \mathrm{H}) .{ }^{13} \mathrm{C}$ NMR $(125$ $\mathrm{MHz}$, acetone- $\left.\mathrm{d}_{6}\right)$ : 143.26, 134.65, 134.06, 128.85, 45.97. HRMS $(\mathrm{FAB})^{+} \mathrm{m} / z$ calcd for $\mathrm{C}_{5} \mathrm{H}_{6} \mathrm{O}_{2} \mathrm{~S}_{2} \mathrm{~K}(\mathrm{M}+\mathrm{K})^{+}, 200.9447$; found, 200.9446.

1-(4-methanesulfonyl-phenyl)-ethanone (Table 1, Entry 3). General procedure (a) was followed using $25 \mathrm{mg}(0.05 \mathrm{mmol})(\mathrm{CuOTf})_{2} \mathrm{PhH}, 123 \mathrm{mg}(1.20 \mathrm{mmol})$ methanesulfinic acid, sodium salt, $11 \mathrm{~L}(0.10 \mathrm{mmol}) N, N^{\prime}$-dimethylethylenediamine, and $246 \mathrm{mg}(1.00$ mmol) 4-iodoacetophenone. The title compound (144 mg, 73\%) was obtained as a light orange solid. M.P. $124{ }^{\circ} \mathrm{C}$ (lit. $\left.{ }^{3} 127{ }^{\circ} \mathrm{C}\right) .{ }^{1} \mathrm{H}$ NMR $\left(500 \mathrm{MHz}\right.$, acetone-d $\left.\mathrm{d}_{6}\right): \quad 8.24$ (d, 2H, J $=6.6 \mathrm{~Hz}), 8.11(\mathrm{~d}, 2 \mathrm{H}, 6.6 \mathrm{~Hz}), 3.21(\mathrm{~s}, 3 \mathrm{H}), 2.70(\mathrm{~s}, 3 \mathrm{H}) .{ }^{13} \mathrm{C}$ NMR $(125 \mathrm{MHz}$, acetone$\left.\mathrm{d}_{6}\right): 197.41,145.66,141.70,129.82,128.46,43.98,27.04$.

\footnotetext{
${ }^{1}$ Otto, R.; Passing, A.; Troeger, J. J. Prekt. Chem. 1893, 47, 102.

${ }^{2}$ Burton, H.; Davy, W. A. J. Chem. Soc. 1948, 525.

${ }^{3}$ Leclerc, G.; Mann, A.; Wermuth, C-G. J. Med. Chem. 1977, 20, 1657.
} 
1-methanesulfonyl-2-methyl-benzene ${ }^{4}$ (Table 1, Entry 4). General procedure (b) was followed using $25 \mathrm{mg}(0.05 \mathrm{mmol})(\mathrm{CuOTf})_{2} \mathrm{PhH}, 123 \mathrm{mg}(1.20 \mathrm{mmol})$ methanesulfinic acid, sodium salt, 11 L (0.10 mmol) $N, N^{\prime}$-dimethylethylenediamine, and 127 L (1.00 mmol) 2-iodotoluene. The title compound (129 mg, 76\%) was obtained as an oil. ${ }^{1} \mathrm{H}$ NMR (500 MHz, acetone- $\left.\mathrm{d}_{6}\right): 7.99(\mathrm{~d}, 1 \mathrm{H}, \mathrm{J}=8.0 \mathrm{~Hz}), 7.62(\mathrm{t}, 1 \mathrm{H}, \mathrm{J}=7.5 \mathrm{~Hz}), 7.48$ (t, $1 \mathrm{H}, \mathrm{J}=6.6 \mathrm{~Hz}), 7.46(\mathrm{~d}, 1 \mathrm{H}, \mathrm{J}=7.1 \mathrm{~Hz}), 3.15(\mathrm{~s}, 3 \mathrm{H}), 2.72(\mathrm{~s}, 3 \mathrm{H}) .{ }^{13} \mathrm{C} \mathrm{NMR}(125 \mathrm{MHz}$, acetone- $\left.\mathrm{d}_{6}\right): 140.24,138.36,134.30,133.46,129.59,127.39,43.70,20.13$. MS (APCI) ${ }^{+}$ $\mathrm{m} / \mathrm{z}$ (rel intens) $171(\mathrm{M}+\mathrm{H}, 100), 91\left(\mathrm{M}-\mathrm{SO}_{2} \mathrm{Me}, 80\right)$.

2-methanesulfonyl-biphenyl (Table 1, Entry 5). General procedure (b) was followed using $25 \mathrm{mg}(0.05 \mathrm{mmol})(\mathrm{CuOTf})_{2} \mathrm{PhH}, 123 \mathrm{mg}(1.20 \mathrm{mmol})$ methanesulfinic acid, sodium salt, $11 \mathrm{~L}(0.10 \mathrm{mmol}) N, N^{\prime}$-dimethylethylenediamine, and $177 \mathrm{~L}$ (1.00 mmol) 2-iodobiphenyl, except the reaction time was $64 \mathrm{~h}$. The title compound (173 $\mathrm{mg}, 75 \%$ ) was obtained as a white solid after purification by flash column chromatography eluted with 3:1 hexanes:ethyl acetate. M.P. $101{ }^{\circ} \mathrm{C}$ (lit. ${ }^{5}$ 100-101 $\left.{ }^{\circ} \mathrm{C}\right) .{ }^{1} \mathrm{H}$ NMR (500 MHz, acetone- $\left.\mathrm{d}_{6}\right): \quad 8.19(\mathrm{dd}, 1 \mathrm{H}, \mathrm{J}=8.0,1.3 \mathrm{~Hz}), 7.78(\mathrm{td}, 1 \mathrm{H}, \mathrm{J}=7.5,1.3 \mathrm{~Hz}), 7.68(\mathrm{td}, 1 \mathrm{H}, \mathrm{J}$ $=7.9,1.4 \mathrm{~Hz}), 7.47-7.52(\mathrm{~m}, 5 \mathrm{H}), 7.44(\mathrm{dd}, 1 \mathrm{H}, \mathrm{J}=7.5,1.2 \mathrm{~Hz}), 2.70(\mathrm{~s}, 3 \mathrm{H}) .{ }^{13} \mathrm{C} \mathrm{NMR}$ (125 MHz, acetone- $\left.\mathrm{d}_{6}\right): 140.24,138.36,134.30,133.46,129.59,127.39,43.70,20.13$. $\mathrm{MS}(\mathrm{APCI})^{+} \mathrm{m} / \mathrm{z}$ (rel intens) $233(\mathrm{M}+\mathrm{H}, 100), 154\left(\mathrm{M}-\mathrm{SO}_{2} \mathrm{Me}, 65\right)$.

1-methanesulfonyl-3,5-dimethyl-benzene (Table 1, Entry 6). General procedure (b) was followed using $25 \mathrm{mg}(0.05 \mathrm{mmol})(\mathrm{CuOTf})_{2} \mathrm{PhH}, 123 \mathrm{mg}(1.20 \mathrm{mmol})$ methanesulfinic acid, sodium salt, $11 \mathrm{~L}(0.10 \mathrm{mmol}) \mathrm{N}, N^{\prime}$-dimethylethylenediamine, and $144 \mathrm{~L}$ (1.00 mmol) 3,5-dimethyliodobenzene. The title compound (167 $\mathrm{mg}, 91 \%$ )

\footnotetext{
${ }^{4}$ Hyatt, J. A.; White, A. W. Synthesis, 1984, 214.

${ }^{5}$ Emrick, D. D.; Truce, W. E. J. Org. Chem. 1960, 25, 1103.
} 
was obtained as a white solid. M.P. $83{ }^{\circ} \mathrm{C}$ (lit. ${ }^{6}$ 81-82.5 $\left.{ }^{\circ} \mathrm{C}\right) .{ }^{1} \mathrm{H}$ NMR $(500 \mathrm{MHz}$, acetone- $\left.\mathrm{d}_{6}\right): \quad 7.57(\mathrm{~s}, 2 \mathrm{H}), 7.38(\mathrm{~s}, 1 \mathrm{H}), 3.09(\mathrm{~s}, 3 \mathrm{H}), 2.43(\mathrm{~s}, 6 \mathrm{H}) .{ }^{13} \mathrm{C}$ NMR $(125 \mathrm{MHz}$, acetone- $\left.\mathrm{d}_{6}\right): 142.10,140.08,135.57,125.38,44.25,21.06 . \mathrm{MS}_{(\mathrm{APCI}}{ }^{+} \mathrm{m} / \mathrm{z}$ (rel intens) $185(\mathrm{M}+\mathrm{H}, 70), 106\left(\mathrm{M}-\mathrm{SO}_{2} \mathrm{Me}, 100\right)$.

1-methanesulfonyl-4-nitro-benzene (Table 1, Entry 7). General procedure (a) was followed using $25 \mathrm{mg}(0.05 \mathrm{mmol})(\mathrm{CuOTf})_{2} \mathrm{PhH}, 123 \mathrm{mg}(1.20 \mathrm{mmol})$ methanesulfinic acid, sodium salt, $11 \mathrm{~L}(0.10 \mathrm{mmol}) N, N^{\prime}$-dimethylethylenediamine, and $249 \mathrm{mg}$ (1.00 mmol) 4-nitroiodobenzene. The title compound (171 $\mathrm{mg}, 85 \%)$ was obtained as a yellow solid. M.P. $125{ }^{\circ} \mathrm{C} .{ }^{1} \mathrm{H}$ NMR (500 MHz, acetone- $\left.\mathrm{d}_{6}\right)$ : $8.52(\mathrm{~d}, 2 \mathrm{H}, \mathrm{J}=8.8 \mathrm{~Hz}), 8.28$ (d, $2 \mathrm{H}, 8.8 \mathrm{~Hz}), 3.29(\mathrm{~s}, 3 \mathrm{H}) .{ }^{13} \mathrm{C}$ NMR (125 MHz, acetone-d $\left.)_{6}\right): 147.30,129.87,129.10$, 125.32, 43.87. Note: The product was obtained as a 12:1 mixture of the desired product and 1,4-bis(methanesulfonyl)-benzene, as determined by ${ }^{1} \mathrm{H}$ NMR: $\quad 8.25$ (s, 4H), 3.26 $(\mathrm{s}, 6 \mathrm{H})$. HRMS $(\mathrm{FAB})^{+} \mathrm{m} / z$ calcd for $\mathrm{C}_{7} \mathrm{H}_{8} \mathrm{NO}_{2} \mathrm{~S}(\mathrm{M}+\mathrm{H})^{+}, 202.0174$; found, 202.0174 .

1-methanesulfonyl-4-methoxy-benzene (Table 1, Entry 8). General procedure (a) was followed using $25 \mathrm{mg}(0.05 \mathrm{mmol})(\mathrm{CuOTf})_{2} \mathrm{PhH}, 123 \mathrm{mg}(1.20 \mathrm{mmol})$ methanesulfinic acid, sodium salt, $11 \mathrm{~L}(0.10 \mathrm{mmol}) N, N^{\prime}$-dimethylethylenediamine, and $234 \mathrm{mg}$ (1.00 mmol) 4-iodoanisole. The title compound (161 mg, 86\%) was obtained as a white solid. M.P. $120{ }^{\circ} \mathrm{C}$ (lit. $\left.{ }^{7} 120{ }^{\circ} \mathrm{C}\right) .{ }^{1} \mathrm{H}$ NMR (500 MHz, acetone-d $\left.\mathrm{d}_{6}\right)$ : 7.89 (d, 2H, J = 8.9 Hz), $7.16(\mathrm{~d}, 2 \mathrm{H}, \mathrm{J}=8.9 \mathrm{~Hz}), 3.94(\mathrm{~s}, 3 \mathrm{H}), 3.08$ (s, 3H). ${ }^{13} \mathrm{C}$ NMR (125 MHz, acetone-d 6 ): 164.44, 134.06, 130.27, 115.20, 56.14, 44.63. MS (APCI) ${ }^{+} \mathrm{m} / \mathrm{z}$ (rel intens) $187(\mathrm{M}+\mathrm{H}$, 30), $108\left(\mathrm{M}-\mathrm{SO}_{2} \mathrm{Me}, 100\right), 78$ (20).

\footnotetext{
${ }^{6}$ Koptyug, V. A.; Gerasimova, T. N. J. Gen. Chem. USSR(Eng. Trans), 1962, 32, 3718.

${ }^{7}$ Suter, C. M.; Hansen, H. L. J. Am. Chem. Soc. 1932, 54, 4101.
} 
1-methanesulfonyl-4-trifluoromethyl-benzene (Table 1, Entry 9). General procedure (b) was followed using $25 \mathrm{mg}(0.05 \mathrm{mmol})(\mathrm{CuOTf})_{2} \mathrm{PhH}, 123 \mathrm{mg}(1.20 \mathrm{mmol})$ methanesulfinic acid, sodium salt, $11 \mathrm{~L}(0.10 \mathrm{mmol}) N, N^{\prime}$-dimethylethylenediamine, and $147 \mathrm{~L}$ (1.00 mmol) 4-trifluoromethyliodobenzene. The title compound (187 mg, $83 \%$ ) was obtained as a white solid. M.P. $103-104{ }^{\circ} \mathrm{C} .{ }^{1} \mathrm{H}$ NMR $\left(500 \mathrm{MHz}\right.$, acetone- $\left.\mathrm{d}_{6}\right)$ :

$8.22(\mathrm{~d}, 2 \mathrm{H}, \mathrm{J}=8.2 \mathrm{~Hz}), 8.05$ (d, 2H, J $8.2 \mathrm{~Hz}), 3.25$ (s, 3H). ${ }^{13} \mathrm{C}$ NMR $(125 \mathrm{MHz}$, acetone- $\mathrm{d}_{6}$ ): 145.83, 135.46, 135.20, 134.94, 134.68, 129.14, 127.64, 127,32, 127.29, 127.26, 127.23, 125.48, 123.31, 121.15, 43.95. HRMS (FAB) ${ }^{+} \mathrm{m} / z$ calcd for $\mathrm{C}_{8} \mathrm{H}_{8} \mathrm{~F}_{3} \mathrm{O}_{2} \mathrm{~S}$ $(\mathrm{M}+\mathrm{H})^{+}, 225.01975$; found, 225.01971.

1-methanesulfonyl-4-methylsulfanyl-benzene (Table 1, Entry 10). General procedure (a) was followed using $25 \mathrm{mg}(0.05 \mathrm{mmol})(\mathrm{CuOTf})_{2} \mathrm{PhH}, 123 \mathrm{mg}(1.20 \mathrm{mmol})$ methanesulfinic acid, sodium salt, $11 \mathrm{~L}(0.10 \mathrm{mmol}) N, N^{\prime}$-dimethylethylenediamine, and $250 \mathrm{mg}$ (1.00 mmol) 4-iodothioanisole. The title compound (181 $\mathrm{mg}, 90 \%)$ was obtained as a white solid. M.P. $98{ }^{\circ} \mathrm{C}$ (lit. $\left.{ }^{8} 98-99{ }^{\circ} \mathrm{C}\right) .{ }^{1} \mathrm{H}$ NMR $\left(500 \mathrm{MHz}\right.$, acetone- $\left.\mathrm{d}_{6}\right)$ : $7.85(\mathrm{~d}, 2 \mathrm{H}, \mathrm{J}=8.1 \mathrm{~Hz}), 7.50(\mathrm{~d}, 2 \mathrm{H}, \mathrm{J}=8.1 \mathrm{~Hz}), 3.11(\mathrm{~s}, 3 \mathrm{H}), 2.61(\mathrm{~s}, 3 \mathrm{H}) .{ }^{13} \mathrm{C} \mathrm{NMR}$ $\left(125 \mathrm{MHz}\right.$, acetone- $\left.\mathrm{d}_{6}\right): 147.51,137.90,128.40,126.10,44.38,14.46 . \mathrm{MS}(\mathrm{APCI})^{+} \mathrm{m} / \mathrm{z}$ (rel intens) $203(\mathrm{M}+\mathrm{H}, 40), 124\left(\mathrm{M}-\mathrm{SO}_{2} \mathrm{Me}, 100\right)$.

1-chloro-4-methanesulfonyl-benzene (Table 1, Entry 11). General procedure (a) was followed using $25 \mathrm{mg}(0.05 \mathrm{mmol})(\mathrm{CuOTf})_{2} \mathrm{PhH}, 123 \mathrm{mg}(1.20 \mathrm{mmol})$ methanesulfinic acid, sodium salt, $11 \mathrm{~L}(0.10 \mathrm{mmol}) N, N$ '-dimethylethylenediamine, and $238 \mathrm{mg}(1.00$ mmol) 4-chloroiodobenzene. The title compound (148 mg, 78\%) was obtained as a white solid. M.P. $96{ }^{\circ} \mathrm{C}$ (lit..$\left.^{9} 98{ }^{\circ} \mathrm{C}\right) . \quad{ }^{1} \mathrm{H}$ NMR $\left(500 \mathrm{MHz}\right.$, acetone- $\left.\mathrm{d}_{6}\right)$ : 7.99 (d, 2H, J = 8.6 $\mathrm{Hz}), 7.72(\mathrm{~d}, 2 \mathrm{H}, \mathrm{J}=8.6 \mathrm{~Hz}), 3.18(\mathrm{~s}, 3 \mathrm{H}) .{ }^{13} \mathrm{C}$ NMR (125 MHz, acetone- $\left.\mathrm{d}_{6}\right)$ : 140.91,

\footnotetext{
${ }^{8}$ Burton, H.; Hu, P. F. J. Chem. Soc. 1948, 604.

${ }^{9}$ Gilbert, E. E. J. Org. Chem. 1963, 28, 1945.
} 
140.04, 130.26, 129.95, 44.17. HRMS $(\mathrm{FAB})^{+} \mathrm{m} / z$ calcd for $\mathrm{C}_{7} \mathrm{H}_{7} \mathrm{O}_{2} \mathrm{SClK}(\mathrm{M}+\mathrm{K})^{+}$, 228.9491; found, 228.9492.

Note: The product was obtained as a $24: 1$ mixture of the desired product and 1,4bis(methanesulfonyl)-benzene, as determined by ${ }^{1} \mathrm{H}$ NMR: $\quad 8.25$ (s, 4H), $3.26(\mathrm{~s}, 6 \mathrm{H})$.

$N$-(4-methanesulfonyl-phenyl)-acetamide (Table 1, Entry 12). General procedure (a) was followed using $25 \mathrm{mg}(0.05 \mathrm{mmol})(\mathrm{CuOTf})_{2} \mathrm{PhH}, 204 \mathrm{mg}(2.00 \mathrm{mmol})$ methanesulfinic acid, sodium salt, $11 \mathrm{~L}(0.10 \mathrm{mmol}) N, N^{\prime}$-dimethylethylenediamine, and $261 \mathrm{mg}(1.00 \mathrm{mmol})$ 4-iodoacetanilide. The crude product was stirred as a suspension in $10 \mathrm{~mL}$ of a 1:1 mixture of ethyl acetate and hexanes for approximately 30 min, and the title compound (58 $\mathrm{mg}, 27 \%$ ) was obtained by filtration as a white solid. M.P. $184{ }^{\circ} \mathrm{C}$ (lit. $\left.{ }^{10} 183-184{ }^{\circ} \mathrm{C}\right) . \quad{ }^{1} \mathrm{H}$ NMR $\left(500 \mathrm{MHz}\right.$, acetone- $\mathrm{d}_{6}$ ): 9.58 (s, broad, $1 \mathrm{H}$ ), $7.89(\mathrm{~d}, 2 \mathrm{H}, \mathrm{J}=9.1 \mathrm{~Hz}), 7.87(\mathrm{~d}, 1 \mathrm{H}, \mathrm{J}=9.1 \mathrm{~Hz}), 3.09$ (s, 3H), 2.15 (s, 3H). ${ }^{13} \mathrm{C} \mathrm{NMR}$ (125 MHz, acetone- $\left.\mathrm{d}_{6}\right): 169.65,144.88,136.00,129.22,119.55,44.49,24.35$. MS $(\mathrm{APCI})^{+} \mathrm{m} / \mathrm{z}$ (rel intens) $214(\mathrm{M}+\mathrm{H}, 35), 93$ (100).

1-methanesulfonyl-naphthalene (Table 1, Entry 13). General procedure (b) was followed using $25 \mathrm{mg}(0.05 \mathrm{mmol})(\mathrm{CuOTf})_{2} \mathrm{PhH}, 123 \mathrm{mg}(1.20 \mathrm{mmol})$ methanesulfinic acid, sodium salt, 11 L (0.10 mmol) $N, N^{\prime}$-dimethylethylenediamine, and 146 L (1.00 mmol) 1-bromonaphthalene. The title compound (50 mg, 24\%) was obtained as a white solid after purification by flash column chromatography eluted with a gradient of 3:1 to 2:1 hexanes:ethyl acetate. ${ }^{1} \mathrm{H}$ NMR $\left(500 \mathrm{MHz}\right.$, acetone- $\left.\mathrm{d}_{6}\right)$ : $8.78(\mathrm{~d}, 1 \mathrm{H}, \mathrm{J}=8.7 \mathrm{~Hz})$, $8.33(\mathrm{t}, 1 \mathrm{H}, \mathrm{J}=4.3 \mathrm{~Hz}), 8.32(\mathrm{~d}, 1 \mathrm{H}, \mathrm{J}=6.3 \mathrm{~Hz}), 8.16(\mathrm{~d}, 1 \mathrm{H}, \mathrm{J}=8.2), 7.80(\mathrm{td}, 1 \mathrm{H}, \mathrm{J}=$ 8.5, 1.4 Hz), 7.73-7.77 (m, 2H), 3.30 (s, 3H). ${ }^{13} \mathrm{C}$ NMR (125 MHz, acetone-d $\left.\mathrm{d}_{6}\right): 137.18$, 135.70, 135.06, 130.07, 130.04, 129.32, 129.26, 127.77, 125.42, 124.91, 44.38.

\footnotetext{
${ }^{10}$ Gilman, H.; Lindblad, A. E. J. Am. Chem. Soc. 1946, 68, 982.
} 
1,1'-sulfonylbis-benzene (Table 2, Entry 1). General procedure (b) was followed using $25 \mathrm{mg}(0.05 \mathrm{mmol})(\mathrm{CuOTf})_{2} \mathrm{PhH}, 263 \mathrm{mg}(1.60 \mathrm{mmol})$ benzenesulfinic acid, sodium salt, $11 \mathrm{~L}(0.10 \mathrm{mmol}) \quad N, N^{\prime}$-dimethylethylenediamine, and $112 \mathrm{~L}(1.00 \mathrm{mmol})$ iodobenzene. The title compound (153 mg, 70\%) was obtained as a white solid. M.P. 125 ${ }^{\circ} \mathrm{C}$ (lit. $\left.{ }^{11} 128-129{ }^{\circ} \mathrm{C}\right) .{ }^{1} \mathrm{H}$ NMR (500 MHz, acetone-D6): 8.01 (m, 4H), 7.68-7.72 (m, 2H), 7.62-7.66 (m, 4H). ${ }^{13} \mathrm{C}$ NMR (125 MHz, acetone-D6): 142.88, 134.17, 130.32, 128.35. MS (APCI) ${ }^{+} \mathrm{m} / \mathrm{z}$ (rel intens) $219(\mathrm{M}+\mathrm{H}, 70), 141(\mathrm{M}-\mathrm{Ph}, 60), 77$ (100).

1,1'-sulfonylbis(4-methyl-benzene) (Table 2, Entry 2). General procedure (a) was followed using $25 \mathrm{mg}(0.05 \mathrm{mmol})(\mathrm{CuOTf})_{2} \mathrm{PhH}, 257 \mathrm{mg}(1.20 \mathrm{mmol}) \quad 4-$ toluenesulfinic acid, sodium salt, $11 \mathrm{~L}(0.10 \mathrm{mmol}) N, N$ '-dimethylethylenediamine, and $218 \mathrm{mg}$ (1.00 mmol) 4-iodotoluene. The title compound (173 mg, 70\%) was obtained as a white solid. M.P. $159{ }^{\circ} \mathrm{C}$ (lit. $\left.{ }^{12} 158^{\circ} \mathrm{C}\right) .{ }^{1} \mathrm{H}$ NMR $\left(500 \mathrm{MHz}\right.$, acetone-d $\left.\mathrm{d}_{6}\right): \quad 7.85$ (d, 4H, J $=8.2 \mathrm{~Hz}), 7.42(\mathrm{~d}, 4 \mathrm{H}, \mathrm{J}=8.2 \mathrm{~Hz}), 2.41(\mathrm{~s}, 6 \mathrm{H}) \cdot{ }^{13} \mathrm{C}$ NMR $\left(125 \mathrm{MHz}\right.$, acetone- $\left.\mathrm{d}_{6}\right)$ : 144.93, 140.43, 130.75, 128.29, 21.34. MS (APCI) ${ }^{+} \mathrm{m} / \mathrm{z}$ (rel intens) $247(\mathrm{M}+\mathrm{H}, 100)$, 155 (M - Tol, 100), 91 (60).

1-methoxy-4-(toluene-4-sulfonyl)-benzene (Table 2, Entry 3). General procedure (a) was followed using $25 \mathrm{mg}(0.05 \mathrm{mmol})(\mathrm{CuOTf})_{2} \mathrm{PhH}, 257 \mathrm{mg}(1.20 \mathrm{mmol}) 4-$ toluenesulfinic acid, sodium salt, $11 \mathrm{~L}(0.10 \mathrm{mmol}) N, N$ '-dimethylethylenediamine, and $234 \mathrm{mg}$ (1.00 mmol) 4-iodoanisole, except the reaction time was $48 \mathrm{~h}$. The title compound (197 mg, 75\%) was obtained as a white solid after purification by flash column chromatography eluted with a gradient of 3:1 to 2:1 hexanes:ethyl acetate. M.P.

\footnotetext{
${ }^{11}$ Sato, K.; Hyodo, M.; Aoki, M.; Zheng, X-Q.; Noyori, R. Tetrahedron, 2001, 57, 2469.

${ }^{12}$ Meyer, H. Ann. 1923, 433, 336.
} 
$105{ }^{\circ} \mathrm{C}$ (lit. $\left.{ }^{13} 103{ }^{\circ} \mathrm{C}\right) .{ }^{1} \mathrm{H}$ NMR $\left(500 \mathrm{MHz}\right.$, acetone- $\left.\mathrm{d}_{6}\right): \quad 7.91(\mathrm{~d}, 2 \mathrm{H}, \mathrm{J}=8.9 \mathrm{~Hz}), 7.84$ $(\mathrm{d}, 2 \mathrm{H}, \mathrm{J}=8.4 \mathrm{~Hz}), 7.41(\mathrm{~d}, 2 \mathrm{H}, \mathrm{J}=8.4 \mathrm{~Hz}), 7.12(\mathrm{~d}, 2 \mathrm{H}, \mathrm{J}=8.9 \mathrm{~Hz}), 3.90(\mathrm{~s}, 3 \mathrm{H}), 2.41$ (s, 3H). ${ }^{13} \mathrm{C}$ NMR (125 MHz, acetone- $\left.\mathrm{d}_{6}\right)$ : 164.25, 144.68, 140.88, 134.77, 130.71, 130.48, 128.09, 115.40, 56.11, 21.32. MS (APCI) ${ }^{+} \mathrm{m} / \mathrm{z}$ (rel intens) $263(\mathrm{M}+\mathrm{H}, 100)$, 171 (M - Tol, 55), 155 (M -PhOMe, 30), 91 (PhOMe, 30).

2-(toluene-4-sulfonyl)-thiophene (Table 2, Entry 4). General procedure (b) was followed using $25 \mathrm{mg}(0.05 \mathrm{mmol})(\mathrm{CuOTf})_{2} \mathrm{PhH}, 257 \mathrm{mg}(1.20 \mathrm{mmol})$ 4toluenesulfinic acid, sodium salt, $11 \mathrm{~L}(0.10 \mathrm{mmol}) N, N$ '-dimethylethylenediamine, and $110 \mathrm{~L}(1.00 \mathrm{mmol})$ 2-iodothiophene. The title compound (103 mg, 43\%) was obtained as a white solid after purification by flash column chromatography eluted with 3:1 hexanes:ethyl acetate. M.P. $120{ }^{\circ} \mathrm{C}$ (lit. $\left.{ }^{14} 113-114{ }^{\circ} \mathrm{C}\right) .{ }^{1} \mathrm{H}$ NMR $\left(500 \mathrm{MHz}\right.$, acetone- $\left.\mathrm{d}_{6}\right)$ : $7.98(\mathrm{dd}, 1 \mathrm{H}, \mathrm{J}=5.0,1.3 \mathrm{~Hz}), 7.91(\mathrm{~d}, 2 \mathrm{H}, \mathrm{J}=8.3 \mathrm{~Hz}), 7.78(\mathrm{dd}, 1 \mathrm{H}, \mathrm{J}=3.8,1.4 \mathrm{~Hz})$, $7.46(\mathrm{~d}, 2 \mathrm{H}, \mathrm{J}=8.3 \mathrm{~Hz}), 7.22(\mathrm{dd}, 1 \mathrm{H}, \mathrm{J}=5.0,3.8 \mathrm{~Hz}), 2.44(\mathrm{~s}, 3 \mathrm{H}) \cdot{ }^{13} \mathrm{C}$ NMR $(125$ MHz, acetone- $\left.\mathrm{d}_{6}\right): 145.26,144.46,140.47,135.28,134.23,130.88,129.01,128.00$, 21.38. $\mathrm{MS}(\mathrm{APCI})^{+} \mathrm{m} / \mathrm{z}$ (rel intens) $238(\mathrm{M}+\mathrm{H}, 90), 155$ ( $\mathrm{M}$ - thiophenyl, 25), 147 (Mtol, 100), 99 (tolyl, 95).

\footnotetext{
${ }^{13}$ Proctor, T.; J. Chem. Soc. 1957, 2312.

${ }^{14}$ Voronkov et al; CHCCAL; Chem. Heterocle. Compd(Eng. Trans.), 1975, 11, 1131.
} 